\title{
Overview of Kidney Transplantation: A Single Centre Report from China
}

\author{
Kiran Jang Kunwar, Li Heng, Fuqing Zeng, Zhendi Wang* \\ Wuhan Union Hospital of China, Tongji Medical College of Huazhong University of Science \& Technology, \\ Wuhan, China \\ Email: kjk_kunwar@hotmail.com, "280460907@qq.com
}

Received 10 March 2016; accepted 2 May 2016; published 5 May 2016

Copyright ( 2016 by authors and Scientific Research Publishing Inc.

This work is licensed under the Creative Commons Attribution International License (CC BY).

http://creativecommons.org/licenses/by/4.0/

(c) ()

\begin{abstract}
Introduction: Worldwide, End Stage Renal Disease (ESRD) is one of the leading disease with prolong morbidity. Kidney transplantation offers the best solution for the problem. The shortage of donor kidney is even bigger problem due to transplantation being one of the routine procedures. The use of deceased donor definitely increases the pool of donor with excellent immediate and long-term follow-up proven results. Aim: The aim is to analyze and summarize the outcome of Kidney transplantation. Methods \& Materials: A total of 78 cases of Kidney Transplantation were selected for the study and categorized as: Group I-41 (living Donor), Group II-23 (DCD) \& Group III-15 (DBD). Perspective study was done with clean data recorded \& maintained pre-operatively, post-operatively and follow-up from Jan 2011 to Dec 2015 in our hospital. Post-operative graft status, complications and at least 1-year follow-up were area of main focus. Results: All patients underwent successful kidney transplantation. In Group I, the number of living donor kidney transplantation is 41 whereas in Group II (DCD) \& III (DBD), the number of deceased donor transplantation is 23 and 15 respectively. The Normal functioning of graft (NGF) was $38(87.8 \%), 16$ (69.6\%) \& $11(73.3 \%)$ in Group I, II \& III respectively along with Poor Graft function (PGF) in Group I-4 (9.7\%), II-5 (21.7\%) \& III-2 (13.3\%) managed by continuing dialysis. Delayed graft function (DGF) was noted I-1 $(2.4 \%)$, II-2 (8.6\%) \& III-1 $(6.6 \%)$ in respective group, which returned to normal function post intervention. Therefore, $1^{\text {st }}$ year graft survival was $>93 \%$ [(Group I (97.6\%), Group II (95.6\%) \& Group III (93.3\%) respectively]. Manageable surgical complication were found in Group I-8 (19.5\%), Group II-5 (21.7\%) \& Group III-2 (13.3\%) like hematoma, hydronephrosis, leakage except one emboli related nephrectomy of transplanted kidney \& one pneumonia led death in Group II. The overall survival was greater than 90\% [(Group I (97.6\%), Group II (91.3\%) \& Group III (93.3\%) respectively] in all three groups after at least 1-year follow-up study, which was an excellent prognosis. Conclusion: Kidney Transplantation is safe, effective and the best method of treatment for ESRD. Significant improvement in quality of life is the hallmark merit over dialysis. Paired donation program should be encouraged in order to over-
\end{abstract}

\footnotetext{
"Corresponding author.
} 
come shortage of kidney, which increases living donor pool. Outcome in living donor Kidney transplantation is always better than deceased donor transplantation. The prognosis of deceased donor transplantation ( 1 year Graft survival $>93 \% \& 1$ year patient survival $>90 \%$ ) is also satisfactory with promising results. Therefore all the results were under acceptable standard limit. Thus, kidney transplantation (live or deceased donor) should be encouraged as primary modalities in the treatment of End Stage Renal Disease (ESRD).

\title{
Keywords
}

\author{
Kidney Transplantation (KTx), ESRD—End Stage Renal Disease, Deceased Donor (DCD—Donor \\ after Cardiac Death, DBD-Donor after Brain Death)
}

\section{Introduction}

End Stage Renal Disease (ESRD) is one of the leading disease worldwide with prolong morbidity. Definite management \& Improvement in quality of life is the benchmark thus, kidney transplantation offers the best solution for the problem [1]. The shortage of donor kidney is even bigger problem due to transplantation service being one of the routine procedures. The use of deceased donor definitely increases the pool of donor with excellent immediate and long-term follow-up proven results [2] [3].

\section{Methods \& Materials}

Prior starting the study, departmental permission followed by approval from the Intuitional Ethics committee was sought. Patient was informed and explained about the merits of the study and consent was taken. All the transplant data were systematically recorded in Chinese Scientific Registry of Kidney Transplantation \& maintained, including medico-legal issues.

\subsection{Study Design}

Patient who underwent kidney transplantation (live donor or deceased donor) from Jan'2011-Dec'2015 were analyzed; thus grouped into Group I (Living donor KTx), Group II (DCD) \& Group III (DBD). A perspective study report was summarized on subjects, which had 1-year minimum follow up amongst transplant recipients. All demographic features like Age, Sex, Creatinine (Pre-operative, Postoperative\& during follow-up), Warm Ischemia Time (WIT), Cold Ischemia Time (CIT) etc were recorded but not outlined in the summary. Post operative graft status, complications and at least 1-year follow up were area of main focus.

NGF: Normal Graft Function; defined as Normal functioning of graft without requirement of any interventions.

PGF: Poor Function of Graft; defined as continuation of dialysis post transplantation.

DGF: Delay Graft Function; defined as requirement of dialysis after first week, post transplantation.

\subsection{Surgery}

Kidney transplant donor surgery was performed with different approach for all three groups: In Group I: Living donor nephrectomy was performed with standard approach \& open donor nephrectomy procedure is our protocol. For Group (II \& III), applications of different surgical approach exist. For Group II (DCD), the following sequence of events was done i.e. process of organ retrieval starts post declaration of cardio pulmonary arrest [Cardiac arrest-Cardiac massage done-Cardiac massage stopped-Death declaration-then Again Starting of Cardiac massage-Perfusion done-Organ procurement-Organ transplantation]. Rapid retrieval of kidney and liver from the cadaver and cooling \& storage is maintained with ice slush. In case of Group III (DBD), the following sequences of events are done: [Brian Death-Catheter insertion-Withdrawal of life support-Death declarationPerfusion started-Organ procurement \& Organ transplantation]. Laparotomy is preferred and post aortic canulation; perfusion with HTK (Histidine-Tryptophan-Ketoglutarate) solution and kidney and liver procurement together has been our approach. Ice slush is used for cooling of kidney \&simple cold storage procedure for storage 
and transportation. To prevent thromboembolism, intravenous 10,000 IU of heparin is routinely given to the donor.

All recipient transplant surgeries were performed with same standard routine technique: Internal iliac \& Renal artery and Renal vein with External Iliac vein anastomosis respectively and ureter anatomized with bladder (extravesical) using modified Lich-Gregoir technique.

\subsection{Immunosuppressant}

The following immunosuppressive regimen was routinely used in every recipient; Induction with Anti thymocyte globulin (ATG) \& steroids; then tapering dose of steroid for maintenance. Calcineurin inhibitors and \& anti-proliferative agents (Mycophenolate Mofetil) has been our drug regimen for transplantation. Drug and doses are varied upon case-by-case basis and adjusted accordingly.

\subsection{Data Analysis}

SPSS software was used to analyze the data.

\section{Results}

All patients underwent successful kidney transplantation. In Group I: 41 cases of living donor kidney transplantation where as in Group (II \& III) deceased donor kidney transplantation was done. Hence, Group II: 23 cases of DCD (donor after Cardiac death \& Group III: 15 cases of DBD (donor after brain death). The Normal functioning of graft (NGF) were 38 (87.8\%), 16 (69.6\%) \& 11 (73.3\%) in Group I, II \& III respectively along with poor graft function (PGF) in Group I-4 (9.7\%), II-5 (21.7\%) \& III-2 (13.3\%)) managed by continuing of dialysis. Delayed graft function (DGF) was also noted I-1 (2.4\%), II-2 (8.6\%) \& III-1 (6.6\%) in respective group, which returned to normal function post intervention. Therefore, $1^{\text {st }}$ year graft survival was $>93 \%$ [(Group I (97.6\%), Group II (95.6\%) \& Group III (93.3\%) respectively].

Manageable surgical complication were found in Group I—8 (19.5\%), Group II—5 (21.7\%) \& Group III—2 (13.3\%) like hematoma, hydronephrosis, leakage except one emboli related nephrectomy of transplanted kidney \& one pneumonia led death in Group II. Any cause of death was also included during follow-up, therefore number of death in Group I-1, II-2 \& III-1 respectively. The overall survival was greater than $90 \%$ [(Group I (97.6\%), Group II (91.3\%) \& Group III (93.3\%) respectively] in all three groups after at least 1-year follow-up study, which is an excellent prognosis. Summary is outlined in Table 1.

\section{Discussion}

With worldwide increase in incidence of diabetes mellitus, glomerulonephritis, hypertension, impaired life style \& its long-term effect has resulted in chronic kidney disease and ultimately to End Stage Renal Disease. ESRD is associated with prolong morbidity and impaired quality of life apart from disease related symptoms [4]. Renal replacement therapy, either with dialysis (Hemodialysis/Peritoneal Dialysis) or kidney transplantation is being the modalities in the treatment for end stage renal disease.

\begin{tabular}{cccc}
\hline Table 1. Transplantation outcome. & & \\
\hline & Live Donor Tx & DCD Tx & DBD Tx \\
\hline Number (n) & 41 & 23 & 15 \\
Normal Graft Function (NGF) & $36(87.8 \%)$ & $16(69.6 \%)$ & $11(73.3 \%)$ \\
Poor Graft Function (PGF) & $4(9.7 \%)$ & $5(21.7 \%)$ & $2(13.3 \%)$ \\
Delayed Graft Non Function (DGF) & $1(2.4 \%)$ & $2(8.6 \%)$ & $1(6.6 \%)$ \\
Surgical Complication & $8(19.5 \%)$ & $5(21.7 \%)$ & $2(13.3 \%)$ \\
1-year Graft Survival & $40(97.6 \%)$ & $22(95.6 \%)$ & $14(93.3 \%)$ \\
1-year Patient Survival & $40(97.6 \%)$ & $21(91.3 \%)$ & $14(93.3 \%)$ \\
\hline
\end{tabular}


Kidney transplantation has been the definite and probably the most satisfactory approach for the treatment of End stage renal disease (ESRD). Prolong morbidity being one of the main reasons behind patient preferences for surgery. Transplant surgery has excelled in last two decade and today kidney transplant surgery is performed as routine procedure in many urology/transplant departments. Scope of kidney transplantation is beyond live donor transplantation. Deceased donor kidney transplantation with donation being from brain death or cardiac death has been routine approach. In fact in many advance centres, kidney transplantation with deceased donor is done more in numbers than live donor. Newly started transplant centre mainly in developing nations usually perform live donor kidney transplantation. With newer immunosuppressant, the rejection rate has declined drastically. The main reason for delay in transplantation is due to organ shortage. Now with law worldwide making deceased donor also an acceptable approach, it has started acting as a major pool for organ donation [5].

In our centre living donor kidney transplantation started from 1977 and being one of the pioneers in kidney transplantation in central china, with New Chinese law regarding deceased donation program; we routinely perform deceased donation (DCD \& DBD) kidney transplantation [6] [7]. Kidney transplantation surgery has been one of the routine procedures in our centre.

The standard approach with minimized Total Ischemia Time (Cold \& Warm) is maintained. Post transplantation, in the first year patient and graft survival rate can be achieved above 90\%. Maximum case series shows the survival rate above $90 \%$ \& in our case (live donor (97.6\%), DCD (91.3\%), DBD (93.3\%) respectively. Manageable surgical complications occurred [Group I (8), II (5) \& III (2)] respectively, which appears acceptable in day-to-day surgery. Studies reveal patient's quality of life improves with quality when compared to patient treated with other modalities [8]-[12]. Definitive treatment \& patient satisfaction is one of the hallmarks of kidney transplantations.

Paired donation program is also another method to overcome shortage of donor pool. Paired donation program should be encouraged in order to overcome shortage of kidney, which increases living donor pool. Surgical complications and post transplantation management will always remain challenging and vital; experience in transplantation has overcome many difficulties. With government verified protocol, our centre also performs paired donation program. Deceased donation kidney transplantation too appears equally effective with long-term satisfactory outcome \& should also be the routine approach apart from live donor approach. Probably deceased donation transplantation will supersede living donor in future. Multi organ retrieval followed by machine perfusion and multi organ transplant should be the usual and standard approach for transplantation [13] [14].

\section{Conclusions}

Kidney Transplantation is safe, effective and the best method of treatment for ESRD. Immediate outcome in living donor Kidney transplantation is better than deceased donor transplantation but the final prognosis (1 year Graft survival $>93 \%$ \& 1 year patient survival $>90 \%$ ) is satisfactory and acceptable in both living and deceased donor kidney transplantation. Significant improvement in quality of life is the hallmark merit over dialysis. Thus, the definitive \& evidence based clinically proven approach for the treatment of End stage renal disease.

Deceased donation kidney transplantation is also an effective approach and should be the routine approach to overcome the huge organ shortage problem. Paired donation program should also be encouraged in order to fulfill the shortage of kidney. Multi organ retrieval and multi organ transplant should be the usual and standard approach for transplantation.

\section{Acknowledgements}

This study was carried out as a part of pure academic interest\& to access the success of our transplant programin the Department of Urology, Wuhan Union Hospital of China, Tongji Medical College, Huazhong University of science \& Technology.

\section{Disclosure}

The authors declare that there is no conflict of interest.

\section{References}

[1] https://en.wikipedia.org/wiki/Kidney_transplantation 
[2] Pine, J.K., Goldsmith, P.J., Ridgway, D.M., et al. (2010) Comparable Outcomes in Donation after Cardiac Death and Donation after Brainstem Death: A Matched Analysis of Renal Transplants. Transplant Proceedings, 42, 3947-3948. http://dx.doi.org/10.1016/j.transproceed.2010.09.072

[3] Ledinh, H., Bonovoisin, C., Weekers, L., et al. (2010) Results of Kidney Transplantation from Donors after Cardiac Death. Transplant Proceedings, 42, 2407-2424. http://dx.doi.org/10.1016/j.transproceed.2010.07.055

[4] http://www.kidneyfund.org/kidney-disease/kidney-failure/

[5] http://www.declarationofistanbul.org

[6] Huang, J., Mao, Y. and Millis, J.M. (2008) Government Policy and Organ Transplantation in China. The Lancet, 372, 1937-1938. http://dx.doi.org/10.1016/S0140-6736(08)61359-8

[7] Chinese Ministry of Health (2011) The Basic Principle of Chinese Human Organ Distribution and Sharing and Core Policy of Liver and Kidney Transplant. Chinese J Transplant, 5, 72-76.

[8] Kim, B.S., Joo, S.H., Ahn, H.J., et al. (2014) Outcomes of Expanded-Criteria Deceased Donor Kidney Transplantation in a Single Centre. Transplantation Proceedings, 46, 1067-1070. http://dx.doi.org/10.1016/j.transproceed.2013.12.014

[9] Kostro, J.Z., Hellman, A., Kobiela, J., et al. (2016) Quality of Life after Kidney Transplantation: A Prospective Study. Transplantation Proceedings, 48, 50-54. http://dx.doi.org/10.1016/j.transproceed.2015.10.058

[10] Rodrigue, J.R., Mandelbrot, D.A., Pavlakis, M., et al. (2010) A Psychological Intervention to Improve Quality of Life and Reduce Psychological Distress in Adults Awaiting Kidney Transplantation. Nephrology Dialysis Transplantation, 26, 709-715. http://dx.doi.org/10.1093/ndt/gfq382

[11] Bura, P. and Bona, M.D. (2006) Quality of Life Following Organ Transplantation. Transplant International, 20, 397409. http://dx.doi.org/10.1111/j.1432-2277.2006.00440.x

[12] Jofre, R., Lopez-Gomez, J.M., Moreno, F., et al. (1998) Changes in Quality of Life after Renal Transplantation. American Journal of Kidney Diseases, 32, 93-100. http://dx.doi.org/10.1053/ajkd.1998.v32.pm9669429

[13] Sedigh, A., Tufveson, G., Backman, L., et al. (2013) Initial Experience with Hypothermic Machine Perfusion of Kidneys from Deceased Donors in the Uppsala Region in Sweden. Transplant Proceedings, 45, 1168-1171. http://dx.doi.org/10.1016/j.transproceed.2012.10.017

[14] Taniguchi, M., Furukawa H., Kawai, T., et al. (2014) Establishment of Educational Program for Multiorgan Procurement from Deceased Donors. Transplant Proceedings, 46, 1071-1073.

http://dx.doi.org/10.1016/j.transproceed.2014.02.002 